\title{
Facial Rehabilitation: A Neuromuscular Reeducation, Patient-Centered Approach
}

\author{
Jessie VanSwearingen, Ph.D., P.T. ${ }^{1,2}$
}

\section{ABSTRACT}

Individuals with facial paralysis and distorted facial expressions and movements secondary to a facial neuromotor disorder experience substantial physical, psychological, and social disability. Previously, facial rehabilitation has not been widely available or considered to be of much benefit. An emerging rehabilitation science of neuromuscular reeducation and evidence for the efficacy of facial neuromuscular reeducation, a process of facilitating the return of intended facial movement patterns and eliminating unwanted patterns of facial movement and expression, may provide patients with disorders of facial paralysis or facial movement control opportunity for the recovery of facial movement and function. We provide a brief overview of the scientific rationale for facial neuromuscular reeducation in the structure and function of the facial neuromotor system, the neuropsychology of facial expression, and relations among expressions, movement, and emotion. The primary purpose is to describe principles of neuromuscular reeducation, assessment and outcome measures, approach to treatment, the process, including surfaceelectromyographic biofeedback as an adjunct to reeducation, and the goal of enhancing the recovery of facial expression and function in a patient-centered approach to facial rehabilitation.

KEYWORDS: Facial rehabilitation, neuromuscular reeducation, facial paralysis

Rehabilitation for facial paralysis or facial movement dysfunction after insult to the facial neuromotor system has previously been of little expected benefit. Thus, the availability of facial rehabilitation is limited, and most individuals with facial movement disorders have been told to await (spontaneous) recovery or told no effective intervention exists. ${ }^{1,2}$ Consequently, individuals with paralysis of or disfiguring facial expressions deal with physical, psychological, and social disability daily. ${ }^{2-7}$ Facial neuromuscular reeducation is a process of facilitating the return of intended facial movement patterns and eliminating unwanted patterns of facial movement and expression. ${ }^{2,3,6,8-14}$ Surface-electromyographic (EMG) biofeedback or mirror feedback serve as adjuncts to the reeducation process, providing accurate information about specific muscles and the timing of activation; the therapist "coaches" patients to (1) enhance facial muscle activity in desired patterns for facial functions and expressions, and (2) reduce (abnormal) facial muscle activity disrupting or preventing intended facial functions. ${ }^{3,8,10,14,15}$ Based on the biological plausibility and gradual but steadily emerging evidence of the efficacy of facial neuromuscular reeducation, ${ }^{8,14,16,17}$ patients with disorders of facial paralysis or facial movement control have an opportunity to explore conservative options for the recovery of facial movement and function.

Characteristics of the facial neuromotor system, an understanding of the neuropsychology of facial

\footnotetext{
${ }^{1}$ Department of Physical Therapy, University of Pittsburgh, Pittsburgh, Pennsylvania; ${ }^{2}$ Facial Nerve Center, University of Pittsburgh Medical Center, Pittsburgh, Pennsylvania.

Address for correspondence and reprint requests: Jessie VanSwearingen, Ph.D., P.T., University of Pittsburgh, Department
}

Facial Paralysis; Guest Editor, Patrick J. Byrne, M.D., F.A.C.S.

Facial Plast Surg 2008;24:250-259. Copyright (C) 2008 by Thieme Medical Publishers, Inc., 333 Seventh Avenue, New York, NY 10001, USA. Tel: +1(212) 584-4662.

DOI 10.1055/s-2008-1075841. ISSN 0736-6825. 
expression, and an intent to map a path that relates specific processes of care of the patient to specific outcomes and to the patient's general health status underlie our focus on facial neuromuscular reeducation and a patient-centered approach to facial rehabilitation. The following sections include (1) a brief description of structure and function of the facial neuromotor system; (2) concepts of the neuropsychology of facial expression and relations among expressions, movement, and emotion; and (3) principles of neuromuscular reeducation in facial rehabilitation to enhance the patient's recovery of facial function, expression, and well-being. An overview of assessment and outcome measures, approach to treatment, some specific treatment options, and the process and goal of facial rehabilitation follow the rationale.

\section{THE FACIAL MOTOR SYSTEM}

The facial motor system is responsible for multiple human functions critical for physical, social, and psychological well-being, such as the physical acts of eating, drinking, and speaking, conversational signals, and even conveying intimate human information. ${ }^{4,7}$ The facial nerve branches and ramifies among nerve fibers within a single bundle of seventh cranial nerve fibers, ${ }^{18}$ a fact that underlies an array of unique spatial patterns of muscle activity and varied synergetic movements demonstrated by individuals with facial nerve disorders. Most often, functional facial movements or expressions are a result of a combination of facial muscle contractions and not the outcome of a single isolated muscle contraction. ${ }^{19}$ For this reason, facial movement is easily distorted by changes in resting facial posture or voluntary movement in any region of the face.

Unlike other skeletal muscles, the facial muscles lack fascial encasement and tendons attaching the facial muscles to bones, thus enabling the origin and insertion of facial muscles to move freely. ${ }^{18}$ The neuromotor control of facial movements also appears different from the usual motor control mechanisms of skeletal muscles due to the limited ability of the facial muscles to provide feedback. Intrinsic muscle receptors and joint receptors, primary sources for peripheral proprioceptive feedback to the central nervous system, are few or absent in the face. ${ }^{20-22}$ With static and dynamic information about facial muscle posture and movement lacking, voluntary attempts to guide facial movements rarely result in an accurate approximation of the desired movement without some sort of compensatory feedback (e.g., mirror) and motor practice (see later).

\section{NEUROPSYCHOLOGY OF FACIAL EXPRESSION}

Facial expressions result from either brain activity involving the motor cortex (cortical behaviors) or by activity of a less clearly defined network of subcortical nuclei and brain-stem areas (subcortical behaviors) ultimately converging on neurons in the facial nuclei. Cortical-mediated expressions are usually voluntary actions with an intended facial function outcome. Subcortical behaviors of the face are usually reactional, an elicited response to previous events, such as surprise, laughter, or sneezing. ${ }^{23}$ Communication or punctuation signals accompanying purposeful language may be a combination of cortical and subcortical behaviors of the face. ${ }^{23,24}$

Highly skilled observers using the Facial Action Coding System distinguish "posed" (e.g., cortical) and "felt" (e.g., subcortical) expressions. ${ }^{24}$ The ability to activate facial muscles, alone, is not sufficient for the complex interactions of facial expressions with human behavior. Electromyographic studies of facial expression associated with emotion indicate that the activity of specific facial muscles change when a person is thinking versus feeling an emotion for individuals with mood disorders. ${ }^{25}$ The dissonance experienced with any attempt to dissociate a patterned facial muscle response from a felt emotion illustrates the intimate association between emotion and facial expression. ${ }^{4,7}$ As emotions elicit a set of stereotyped facial muscle contractions of an expression, the alternative may also be true, facial muscle activity may elicit or reinforce emotions (e.g., facial feedback hypothesis). ${ }^{4,25,26}$ Given the absence of proprioceptive feedback from facial muscles, the mechanism is unclear, yet some clinical evidence supports the concept. Psychological distress mediates the association between impairment and disability in individuals with a facial neuromotor disorder. ${ }^{27}$ The presence of a marker of positive affect predicts greater response to therapy to increase lip corner movement with smiling in individuals with a facial neuromotor disorder. ${ }^{19,28}$ The degree of distortion of the pattern for smiling from the pattern defined for a smile of happiness was associated with greater psychological dysfunction. ${ }^{29,30}$ Evidence supporting the facial feedback hypothesis could significantly impact therapeutic interventions for individuals with distorted or weakened facial movements and the understanding of psychosocial disorders among the patient population.

Little specific information is known about the contribution of deficient facial feedback to the psychosocial problems of individuals with facial dysfunction. ${ }^{31}$ Investigators describing problems related to facial disfigurement emphasized the impact of facial dysfunction on social interactions, including psychological and physical fatigue from managing social interactions (e.g., making interactions "easier" for those who interact with the one with disfigurement). Such issues of the control of facial motor activity of facial expressions have an important bearing on the recovery of facial neuromotor function through rehabilitation after insult to the 
facial motor system. ${ }^{32}$ Training muscle activity underlying specific facial expressions may be more effective or efficacious for true recovery (e.g., improved quality of life) than is training muscle activity for restoration of facial movement (at the impairment level) for the individual with a facial neuromuscular disorder. ${ }^{30,32,33}$ Particularly (1) the pattern of facial muscle activity of an expression may guide the physical therapist in developing tailored treatment strategies using neuromuscular reeducation; (2) emotions or other subcortical-initiated expressions may be valuable adjuncts to treatment of facial neuromotor disorders; and (3) emotional status or mood may influence the facial muscle activity and alter the course and outcome of treatment for individuals with facial nerve disorders.

\section{REHABILITATION FOR FACIAL NEUROMOTOR DISORDERS}

\section{Traditional Approach to Facial Rehabilitation}

Rehabilitation for facial neuromotor disorders through physical therapy using nonspecific light massage, electrical stimulation, and repetitions of common facial expressions in a general exercise regimen have been considered to be of little benefit. ${ }^{34,35}$ In fact, some interventions may even adversely affect recovery of facial neuromotor function. ${ }^{35-37}$ Evidence from animal studies suggest electrical stimulation of facial neuromusculature during recovery from nerve injury may be disruptive to reinnervation $^{37-39}$ (however, see Refs. 40, 41). Some individuals treated with electrical stimulation during rehabilitation of the facial nerve injury have been observed to produce "mass action," a generalized contraction of all or many of the facial muscles with attempted voluntary facial movements or expressions. ${ }^{15,42}$ The use of nonspecific electrical stimulation of the peripheral facial neuromuscular system during the recovery process reinforces abnormal (synkinetic) patterns of facial muscle activity. If the electrical stimulus is not carefully localized to the facial muscle nerve branch serving the specific intended facial movement or expression, peripheral nerve fibers within a nerve trunk serving many facial muscles ${ }^{43}$ will be simultaneously recruited. In our clinical experience, many patients who have had prior electrical stimulation therapy demonstrate facial movements with reinforced synkinetic muscle activity and inaccurate patterns of facial expressions. ${ }^{44}$

\section{Facial Neuromuscular Reeducation Approach to Facial Rehabilitation}

Several investigators have described improvements in facial movement as an outcome of facial neuromuscular reeducation using surface EMG biofeedback or mirror feedback. $^{3,6,8-14,17}$ Facial neuromuscular reeducation is a process of relearning facial movement using specific and accurate feedback to (1) facilitate facial muscle activity in functional patterns of facial movement and expression and (2) suppress abnormal muscle activity interfering with facial function. $3,6,15,45$ To date, few studies have defined either a specific approach to the use of EMG biofeedback or measurable outcomes of the treatment. ${ }^{6,32,44}$

The theoretical benefit of using EMG biofeedback as an adjunct to facial muscle reeducation in the treatment of facial paralysis (little or no movement) or synkinesis (abnormal movement accompanies the intended movement) is the ability to provide the individual accurate and immediate feedback about facial muscle activity. In the case of little or no facial movement, surface EMG provides immediate and accurate information about the patient's attempts to increase facial muscle activity. For patients with abnormal facial movement accompanying and distorting the intended facial action (too much movement), surface EMG provides the information about the patient's attempts to decrease the abnormal muscle activity while maintaining or increasing activity of the muscles underlying the intended facial action. Individuals with intact proprioception (mechanism for feedback) relearn the movements of walking after injury of the lower extremity peripheral neuromuscular system. The absence of feedback intrinsic to the facial muscles presumably renders the brain uninformed of facial muscle performance. Given adequate information about performance for the purpose of motor learning, the facial neuromotor system has the same ability to relearn movement patterns, as the peripheral neuromuscular system of the lower extremity. Individuals who are provided precise, extrinsic feedback about facial muscle activity (e.g., surface EMG) learn to recruit the appropriate motor units for the desired expression and learn which motor units are inappropriate to recruit for the intended task. $3,6,10,14$

\section{Inadequacy of the Description of the Problem, Treatment, and Treatment Outcome}

The evidence for efficacy of neuromuscular reeducation for recovery of facial function and expressions in individuals with a facial neuromotor problem stems from descriptive (case reports or retrospective outcome studies) and a few randomized controlled clinical trials with small sample sizes, with feedback-assisted (surface EMG or mirror or both) neuromuscular reeducation demonstrated to be associated with generally better or equivalent outcomes to the comparison standard of care for patients with facial neuromotor disorders. ${ }^{6,17,44}$ None of the studies clearly defined what patients do well with what treatments, nor did the investigators define an adequate measurement of treatment outcome, including impairment and disability level, patientcentered outcomes. 
A systematic means of defining specific characteristics of the patient's facial neuromotor disorder, particularly impairment and disability problems, and targeting physical therapy in facial rehabilitation for the patient-specific problem areas may improve the delivery and outcomes of facial rehabilitation. ${ }^{46}$ A treatmentbased classification system could be used in the process of further defining facial neuromotor problems ${ }^{47}$ and providing a guide to the appropriate treatment approach. ${ }^{47-49}$ Treatment-based classification also serves as a means of validating specific interventions relative to the effectiveness of treatments matched for a treatment category.

The role of classification in rehabilitation mimics the role of diagnosis in medicine-identification of a clinical problems based on sets of signs and symptoms, for the purpose of applying a known treatment. ${ }^{50,51}$ Multiple pathologies underlie a facial neuromotor disorder (e.g., Bell's palsy, tumors, trauma), ${ }^{51-55}$ yet the pathology provides little information useful in matching the appropriate treatment to the problem. Instead, treatments are matched to the patient's specific signs and symptoms of facial neuromotor dysfunction associated with the pathology. For example, an individual with Bell's palsy could have severe weakness or little weakness but marked synkinesis (abnormal movement). The treatments differ markedly for a primary problem of facial weakness versus a problem of abnormal facial movement, yet the same pathology. In our clinical experience, among patients treated based on physical signs of facial neuromuscular dysfunction and symptoms of difficulties in activities of daily living involving the face, certain treatments got certain patients better.

\section{ASSESSIMENT INTO PRACTICE: DESCRIBING THE PROBLEM AND RECOGNIZING THE OUTCOME}

A clinical practice and research goal in the rehabilitation of facial neuromotor disorders is to trace a path that relates specific processes of care of the patient to specific outcomes to the patient's general health status. ${ }^{56}$ Using the International Classification of Impairments, Disabilities and Handicaps (ICIDH) developed by the World Health Organization (WHO) as a conceptual framework, ${ }^{49,57}$ we defined assessment measures in domains of impairment, disability, and aspects of healthrelated quality of life (HRQL) useful in assessment and determining outcomes of treatment. The Facial Grading System $^{58}$ measure of facial impairment and the Facial Disability Index ${ }^{59}$ measure of disability, with other factors of the illness, combined accounted for $72 \%$ of the variance (impairment, 28.64\%, disability, 25.17\%, and temporal characteristics of disease, $18.16 \%$ ) in describing patients with a facial neuromotor disorder. ${ }^{46}$

\section{Impairment Testing}

The Facial Grading System (FGS) is a performancebased measure ${ }^{58}$ of facial impairment in three areas: (1) resting posture of the eye, the nasolabial (cheek) fold, and the corner of the mouth; (2) voluntary movement for five expressions in five regions of the face, forehead wrinkles, eye closure, open mouth smile, snarl, and pucker; and (3) synkinesis, associated with the voluntary movement tests. The psychometric properties of the FGS have been defined, including construct validity and responsiveness of the FGS for clinically meaningful change for patients with a facial neuromotor disorder, ${ }^{58}$ and interrater and intrarater reliability for scale use in assessment of patients with facial neuromotor disorders (reliability coefficients $\geq 0.90$ ). ${ }^{46}$

\section{Measuring Facial Disability}

The Facial Disability Index (FDI) is a disease-specific, self-report instrument for the assessment of the disabilities of patients with facial nerve disorders, scored as two subscales: the FDI physical and FDI social subscales. ${ }^{59}$ Reliability ${ }^{59}$ and construct validity of the FDI subscales has been demonstrated by correlation with clinical measures of facial movement and psychosocial status and by comparison with the Short Form $-36^{60}$ general HRQL measure. ${ }^{59}$

\section{Treatment-Based Classification Categories}

Based on physical signs and symptoms identified by using the FGS impairment and FDI disability measures, we classify patients with facial neuromotor disorders into one of four treatment-based categories (initiation, facilitation, movement control, and relaxation). ${ }^{47}$ The physical therapy approach (suggested by the name of the treatment category) specifically targeted for the set of signs and symptoms of each category are based on the biological plausibility that the underlying neuromuscular physiology differed by treatment category.

\section{INITIATION}

In the initiation category, patients typically demonstrate moderate to marked asymmetry of the face at rest (e.g., a drooped face, including lower eyelid, depressed cheek, and drooped mouth corner) and marked asymmetry with voluntary movement or spontaneous expressions or functions (e.g., little or no ability to initiate movement on the involved side), without abnormal movements (synkinesis). Activity of the uninvolved facial musculature, in the presence of the profound involved side facial weakness, may actually shift the face toward the uninvolved side with facial functions and expressions. Facial functions, such as closing the eye, eating and drinking, speaking, or rinsing the mouth are most difficult for patients in this category. The appearance of obvious 
facial disfigurement at rest and with movement has a major negative impact on psychosocial well-being and daily interpersonal interactions.

Treatment specific for patients in the initiation category includes active assisted range of motion exercises and small range movement practice to avoid overpowering by the muscle function of the uninvolved side of the face. Describing the usual process of recovery and rehabilitation and expected signs of recovery of facial movement and function also seems to be beneficial.

\section{FACILITATION}

Mild to moderate facial asymmetry at rest (e.g., slight lower eyelid droop, minimal flattening of the cheek fold, and minimal droop of the corner of the mouth), ability to initiate facial muscle activity, but mild to moderate asymmetry of the face with attempted voluntary movement and with expressions (e.g., incomplete facial functions and expressions), and typically no or little synkinesis characterize the presentation of patients in the facilitation category. Primary movement problems include insufficient protection of the eye, secondary to difficulty closing or sustaining eye closure for moistening and protection on a windy day or when washing the face, and mild but notable problems eating, drinking, and rinsing the mouth, without loss of food or fluid (essentially a milder version of the initiation category problems). Psychosocial distress is typically less than for initiation, mirroring the lesser severity of resting and voluntary facial asymmetry and physical disabilities.

The patients in the facilitation category initiate facial movement, so active and resistive exercises to increase facial movement excursion are appropriate. Education includes emphasizing the importance of accurate exercise practice over quantity and an awareness of signs of some typical abnormal movement patterns (synkinesis) that may develop with the increasing movement. The recognition of any synkinetic movement may indicate the need to return for a therapy visit to avoid continued exercise with synkinesis reinforcing undesirable patterns of facial movement.

\section{MOVEMENT CONTROL}

Most characterized by asymmetry, some at rest, and usually more obvious with movement, the asymmetry characteristic of patients in the movement category is not "droop" but tightening or retraction of the face. A narrowed aperture between the eyelids (usually lower eyelid raised), a deepened cheek fold, and sometimes puffy or plumped cheek appearance, with or without a lateral and upward pull on the corner of the mouth, is characteristic, and the center of the lips (philtrum) may be shifted laterally toward the involved side of the face. Weakness as for the facilitation category is common, but the key sign is the unintended movement of one region of the face, associated with an intended facial movement or expression, synkinesis (e.g., eye closure with smile, and retraction of the corner of the mouth and deepening of the cheek fold with a raise of the brow). Though the reason for the problem differs for the movement control compared with the facilitation category, the physical problems are similar arising largely from the abnormal movement patterns, not weakness. Patients report excessive tearing of the eye, difficulty keeping the eye open when speaking or eating, biting the inside of the cheek when eating, and speech problems when speaking quickly. The synkinetic movements, not intended yet accompanying every performance of the associated intended facial movement, function, or expression, seriously disrupt personal and work relations, and the patients with movement control problems frequently report moderate to marked psychosocial distress.

Facial neuromuscular reeducation for the problems of facial movement control involve guiding the patient in learning (relearning) to isolate muscle contractions and reduce the muscle activity of abnormal patterns of movement. With a short-term treatment goal of producing desired facial movements or expression patterns without the accompanying synkinetic movement, some therapists recommend small movement therapy. $8,42,44$ However, facilitating the desired movement pattern (and increasing facial muscle activity of the intended movement) and accepting some but minimal synkinetic movement in the process may also be effective in the recovery process. ${ }^{14}$ The presumed process of reorganization in the brain to learn to recruit the appropriate neurons to increase facial movement suppresses the recruitment of neurons for the synkinetic movement. As the patient learns appropriate patterns of movement control, the patient may be reclassified into the facilitation category for continued treatment and recovery. Stretching exercises are also indicated to lengthen facial muscle tissues shortened secondary to abnormal patterns of movement and even facial muscle guarding, as patients try to restrict all movement to avoid the synkinesis and disfiguring movement.

\section{RELAXATION}

Characteristic of the relaxation treatment category is a combination of marked asymmetry of facial posture at rest (as for the movement control category), with spontaneous twitching and facial muscle spasms. The facial muscle spasms often increase in frequency and amplitude with greater movement effort. Muscle strength is typically not the major problem, yet the observed facial movement may be moderately restricted as the patient guards against the "next spasm." Specifically, uncontrolled orbicularis oculi spasms close the eye unintentionally and make some instrumental activities of daily living unsafe and others such as hobbies too fatiguing to continue. Psychosocial problems are common and marked, as individuals with facial twitches and spasms 
spend much energy and time controlling facial movement and worrying that the spasm can or will return, interfering with the activities of the day.

The primary treatment goal for problems of facial twitches and spasms is relaxation exercises, such as modifications of the standard relaxation exercises originally described by Jacobson ${ }^{61}$ and small rhythmic, alternating movements to relax the muscles. Techniques to inhibit muscle activation include sustained stretching and cross-friction massage to reduce passive tissue restrictions.

\section{DIFFICULT CHALLENGES IN REEDUCATION FOR FACIAL MOVEMENT DISORDERS}

Restoring symmetry to facial movements, particularly in spontaneous facial expressions and functions characteristic of everyday facial actions and not practiced, voluntarily guided facial movements such as posing for a photo or to please the therapist, represents one of the most difficult challenges in facial rehabilitation. The lack of symmetry in movement of the face may be due to weak muscles unable to produce adequate force to participate equally with the muscles of the uninvolved side of the face. Asymmetry may also be secondary to abnormal movement of the involved side restricting or distorting facial muscle activity of the uninvolved side because of a mismatch in force, speed, and amplitude of facial movement side to side. A compensatory or perhaps overcompensation by muscles of the uninvolved side of the face may eliminate a need for the involved side action, redefining the muscle pattern for the task or rendering the involved side muscle less effective or ineffective because of timing delays or altered position or length of the involved side muscle prior to or during the desired facial action. Such asymmetrical facial movements, most often recognized as distortion of the face during movement (e.g., talks out of one side of the mouth) may develop and persist, in part because of the lack of proprioceptive feedback to inform the brain of the errors in facial movement patterns, and because the facial action subserved by the asymmetrical pattern of facial movement is necessary and common, thus practiced in the distorted fashion repeatedly throughout the day.

The example, "talks out of one side of the mouth" is common for patients in the initiation category, and the maladaptive pattern of facial movement develops and gets practiced because speech is necessary. With profound weakness, movements of the lips may be impossible, yet talking must go on! The brain meets the challenge of intelligible speech by altering the muscle activation pattern to bring the lips together at any point in space (and any side of the face) to produce the intended speech sounds. Thus, speaking, may promote asymmetrical facial movement patterns. Even with the reinnervation, rehabilitation, and recovery of facial muscle activity on the involved side of the face, without the brain recognizing the muscle pattern for speech is altered (lack of feedback from muscles), the distorted pattern likely persists.

Repeated attempts to smile as much as possible to rehabilitate the smiling muscles on the involved side may be a specific and effective conditioning program for the uninvolved smiling muscles. Unfortunately, the repeated attempts to smile as much as possible may do little to activate the involved-side smiling muscles. In fact, an abnormal lengthening of the partially or completely paralyzed involved-side smiling muscles may result in part from the impact of repeated smiling attempts on the involved-side smiling muscles, too weak to oppose or match the pull of the uninvolved facial musculature.

Exercises that tend to center the face so that the center of the lips moves more to the center with the exercise or for which the lip center is shifted little or not at all with the exercise help avoid the problem of abnormal lengthening and enable involved-side muscle motor practice. Several exercises by observation and clinical experience help integrate involved-side muscle activity with the uninvolved, provide opportunities for the involved-side muscle to exercise, particularly in desired patterns of movement, and promote coordination (e.g., simultaneous activation of involved with uninvolved muscles for the facial task). For example: (1) suck the cheeks between the teeth; (2) wrap your lips over the teeth; (3) make the " $\mathrm{f}$ " sound (sustained, "ffffffffff" sound); and (4) blowing, as if blowing bubbles in your drink, through a straw, or blowing bubbles through a bubble wand. The blowing exercise incorporates the benefit of context of the movement to enhance the amplitude and coordination, more so than found for the deliberate, voluntary (e.g., cortically mediated) movement of pucker, despite the fact that both tasks require a similar muscle for the action, the orbicularis oris. ${ }^{33}$ The exercises described for enhancing symmetry are in part or wholly well-learned, semiautomatic (subcortically mediated) facial actions, and most people clearly understand the exercise goal.

The facial centering exercises for integrating and coordinating involved with uninvolved facial muscle activity and enabling the involved side to participate in exercises and facial functions also serve as the basis for exercises to regain mid-cheek lift and a "real" smile. Elevation of the mid-cheek region, a responsibility of the levator labii, superior orbicularis oris, and even the medial aspect of the orbicularis oculi muscles, accompanies many facial expressions and functions, such as smiling, speech sounds (e.g., sh, f, b, p . . ), sniffling, and facial expressions of disgust and perplexity. In most functions, the role of the mid-cheek muscles remains one of synergist, or quiet but necessary companion to the facial task. Thus, few individuals with a facial neuromotor 
disorder, and perhaps even those without, understand and activate the mid-cheek muscles easily or consistently on voluntary request to do so. And, voluntary attempts to activate the mid-cheek lift muscles frequently result in a wide variety of funny faces, many without mid-cheek muscle activation. Activation of the mid-cheek muscles integrated between the involved and uninvolved side also further serves to reinforce the centering of the face and enhance coordinated movement between the sides of the face. For example: (1) blowing as if blowing bubbles, keep blowing and try to add knit eyebrows together as if thinking; (2) blowing as if blowing bubbles, keep the air blowing, and transition to a "fffff" sound, alternate between blowing and "fffff" trying to keep air moving through the lips; (3) make the "fffff" sound, keep the "fffff" sound and try to add a smile, "from the corner of your eyes"; (4) blowing as if blowing bubbles and add a smile "from the corner of your eyes." The smile "from the corner of your eyes" directive in the above exercises is designed to facilitate the pattern of muscles and timing of muscle activity characteristic of a smile of happiness. ${ }^{4,62-64}$ The Duchenne marker or positive affect marker of a smile of joy or happiness is the activation of the orbicularis oculi muscle with the zygomaticus muscle group (typical smiling muscles) and mid-cheek synergists. Actually, encouraging the individual with a facial movement disorder to blow as if blowing bubbles and then to try to relive (think about) a time when the individual was really happy may be the most effective method for performing the exercise, as reliving happiness tends to activate the positive affect marker, ${ }^{4,62-64}$ the orbicularis oculi contraction in association with smiling.

The recovery of eye closure and related functions of the orbicularis oculi muscle of the eye with the eye represent similarly challenging problems in facial rehabilitation. A major problem is recovery of the exquisite integration of eye movements with eyelid and face movements and the ability to use the appropriate pattern of muscle activation and relaxation for the intended task, most tasks that are largely performed with little or no conscious attention. Gentle eye closure involves relaxation of the levator muscle of the eyelid served by the oculomotor nerve. ${ }^{43}$ (Mueller's muscle, a smooth muscle of the upper eyelid innervated by sympathetic nerve fibers, also participates, but the muscle has not been included here to streamline the discussion.) The relaxation of the levator allows the upper eyelid to descend. A contraction of the superior rectus muscle (extraocular muscle for moving the eyeball), which moves the eyeball in an upward direction, immediately follows the relaxation of the upper lid. ${ }^{65-67}$ Only the brief contraction of the orbicularis oculi inhibits the superior rectus muscle and prevents the upward migration of the eyeball. As a result of the orbicularis oculi contraction timed to inhibit the supe- rior rectus muscle ongoing activation, the eye follows the descent of the eyelid to come to rest in a slightly downward position behind the closed eyelid. Weakness of the orbicularis oculi muscle (innervated by the facial nerve) results in the Bell's phenomenon, commonly observed with eye closure in individuals with a facial neuromotor disorder. The Bell's phenomenon, or the upward movement of the eyeball (superior rectus muscle action uninhibited by the paralyzed orbicularis oculi muscle), prevents or interferes with the descent of the relaxed upper eyelid. ${ }^{67}$ The characteristic incomplete eye closure, inability to effectively blink, eyes open at night when sleeping, and the high risk of drying of the eye and serious damage to the cornea are associated with the disruption of coordinated eye closure. ${ }^{43}$ Even with reinnervation and recovery of facial movement, the Bell's phenomenon pattern may persist. ${ }^{8}$

Several neuromuscular reeducation strategies based on an understanding of the physiologic pattern of muscle actions for eye closure have been implemented to overcome the Bell's phenomenon barrier to usual eye functions and facilitate eye closure. One strategy, eye closure while looking down, is an attempt to relax the superior rectus muscle of the eye. The exercise instructions for the sequential steps of the exercise, "look down, close eyes, once closed continue to look down," aim to voluntarily reduce the tendency of Bell's phenomenon to lead to sustained superior rectus activation and difficulty closing. A second strategy, more appropriate when the patient has some return of orbicularis oculi muscle activation, relies on voluntary activation of the orbicularis oculi to assist in relaxing the superior rectus activation. The sequential exercise instructions, "squint, look down, and close eyes, then relax squint," is intended to activate the orbicularis oculi, add relaxation of the superior rectus (look down), and then follow with voluntary relaxation of the orbicularis oculi to achieve the eye closure resting state. $^{68}$

\section{EXERCISE PRACTICE INTO LIFE}

In addition to clinical training sessions, an individualized home facial movement exercise program designed to reinforce the desired facial movement patterns provides the practice component of motor learning necessary for neuromuscular reeducation. Only exercises that the patient demonstrates the ability to perform accurately for the goal of treatment or for which the patient may not consistently perform accurately but recognizes accurate and not so accurate performance and can correct or redo exercise to optimize practice are recommended for the home program. The exercise program usually consists of 5 to 10 repetitions of 3 to 5 exercises to be done twice daily. Use of a mirror for visual feedback during exercise can 
be helpful for the patient, but mirror images, watching oneself move with facial paralysis, and the psychological distress of looking at an abnormal facial appearance may be more detrimental to recovery than beneficial. Striving to design home exercise and train patients during the clinical sessions to perform a couple of exercises accurately without the need for feedback guidance, or with only an occasional need to look in the mirror once the exercise has begun to "check" that the motion or position of the face during the exercise is as desired, has had positive results both in terms of the recovery of facial function and the patient's experience of the process. The success of neuromuscular reeducation, much like any exercise program to enhance motor skill (e.g., learning to play tennis or golf) depends in part on attention and practice, practice, practice to become a highly skilled mover. ${ }^{69}$ Patients to be successful through facial neuromuscular reeducation commit sometimes to tedious thoughtful exercises for a long duration, thus a reason to monitor and care about the patient's experience during rehabilitation. Some usual or not so usual tasks automatically involve facial muscles in movement patterns that enhance facial movement outcomes and may be effective components of the home exercise program; for example, sucking through a straw, attempting to play a harmonica, saying all the words you can think of that begin with the sounds "sh," "f," "p," or "b" with the teeth together (teeth together facilitates lip movements and avoids jaw movement sometimes used to substitute for weak lip movements [personal experience]), and think of a time you were happy and smile every time you pass someone walking on the street. Such exercises may also serve to transition the process of facial rehabilitation into using the face in everyday life.

Instructions for exercises, massage, and repetitions are individualized and written down for patients; the therapist often writing the exercise description as the patient demonstrates exercise performance at the end of the therapeutic session and leaves for one or more weeks of daily, solo practice. As indicated by the signs and symptoms noted in reevaluations at subsequent physical therapy sessions, regular updates to the facial exercise home program are important. Replacing previous exercise regimens with a new set of 3 to 5 exercises is indicated to match the exercises to the current (and evolving) movement problems but also to enhance the patient's interest in the reeducation process and to challenge the brain in learning or relearning the repertoire of facial movements, expressions, and functions.

\section{SIGNIFICANCE OF FACIAL REHABILITATION}

To trace the path that relates specific process of care of the patient with a facial neuromotor disorder to specific patient-centered outcomes and restored or improved health-related quality of life requires a foundation for assessment and treatment, based in biological plausibility and supported by evidence from clinical practice and trials. ${ }^{51,56}$ The use of treatment approaches tailored to the pretreatment classification may result in more costeffective treatment for an underserved clinical population. Second, treatment of facial neuromotor disorders provides a rich and complex integration of the neuroscience of movement control, the linkage of emotion and movement, and the impact of psychosocial factors in the presentation of motor disorders, which may be applicable in understanding movement problems in individuals with other types of neuromotor disorders.

\section{REFERENCES}

1. Bateman DE. Facial palsy. Br J Hosp Med 1992;47:430431

2. Ohye RG, Altenberger EA. Bell's palsy. Am Fam Physician 1989;40:159-166

3. Brudny J, Hammerschlag PE, Cohen NL, et al. Electromyographic rehabilitation of facial function and introduction of a facial paralysis grading scale for hypoglossalfacial nerve anastomosis. Laryngoscope 1988;98:405-410

4. Ekman P. Psychosocial aspects of facial paralysis. In: May M, ed. The Facial Nerve. New York, NY: Thieme Medical Publishers; 1986:781-787

5. Neely JG, Neufeld PS. Defining functional limitation, disability, and societal limitations in patients with facial paresis: initial pilot questionnaire. Am J Otol 1996;17:340-342

6. Ross B, Nedzelski JM, McLean JA. Efficacy of feedback training in longstanding facial nerve paresis. Laryngoscope 1991;101:744-750

7. Twerski AJ, Twerski B. The emotional impact of facial paralysis. In: May M, ed. The Facial Nerve. New York, NY: Thieme Medical Publishers; 1986:788-794

8. Brach JS, VanSwearingen JM, Lenert J, Johnson PC. Facial neuromuscular retraining for oral synkinesis. Plast Reconstr Surg 1997;99:1922-1933

9. Brown DM, Nahai F, Wolf S, et al. Electromyographic biofeedback in the re-education of facial palsy. Am J Phys Med 1978;57:183-190

10. Hammerschlag PE, Brudny J, Cusumano R, et al. Hypoglossal-facial nerve anastomosis and electromyographic feedback rehabilitation. Laryngoscope 1987;97:705-709

11. Jankel WR. Bell's palsy: muscle re-education by electromyographic feedback. Arch Phys Med Rehabil 1978;59:240-242

12. Schram G, Burres S. Nonsurgical rehabilitation after facial paralysis: a 25 year study. In: Portmann M, ed. Facial Nerve. New York, NY: Masson; 1981:461-462

13. Walravens S. Using EMG biofeedback in the treatment of facial paralysis. Acta Otorhinolaryngol Belg 1986;40:174177

14. VanSwearingen JM, Brach JS. Changes in facial movement and synkinesis with facial neuromuscular reeducation. Plast Reconstr Surg 2003;111:2370-2375

15. Diels HJ. New concepts in nonsurgical facial nerve rehabilitation. In: Meyers E, ed. Advances in Otolaryngology Head and Neck Surgery. Chicago, IL: Mosby Year Book, Inc., 1995;9: 289-315 
16. Deleyiannis FW, Akari M, Schmidt KL, Henkelmann TC, VanSwearingen JM, Manders EK. Muscle activity in the partially paralyzed face after placement of a fascial sling. Ann Plast Surg 2005;55:449-455

17. Manikandan N. Effect of facial neuromuscular re-education on facial symmetry in patients with Bell's palsy: a randomized controlled trial. Clin Rehabil 2007;21:338-343

18. Brodal A. Neurological Anatomy in Relation to Clinical Medicine. Third ed. New York, NY: Oxford University Press; 1981

19. Schmidt KL, VanSwearingen JM, Levenstein RM. Speed, amplitude, and asymmetry of lip movement in voluntary puckering and blowing expressions: implications fo facial assessment. Motor Control 2005;9:270-280

20. Baumel JJ. Trigeminal-facial nerve communications. Arch Otolaryngol 1974;99:34-44

21. Brudny J. Biofeedback in facial paralysis: electromyographic rehabilitation. In: Rubin L, ed. The Paralyzed Face. St. Louis, MO: Mosby Yearbook; 1991:247-264

22. Burgess PR, Wei JY, Clark FJ, et al. Signaling of kinesthetic information by peripheral sensory receptors. Annu Rev Neurosci 1982;5:171-187

23. Rinn WE. The neuropsychology of facial expression: a review of the neurological and psychological mechanisms for producing facial expressions. Psychol Bull 1984;95:52-77

24. Ekman P, Friesen WV. Measuring facial movement. Environ Psychol Nonverb Behav. 1976;1:56-75

25. Schwartz GE, Fair PL, Salt $P$, et al. Facial muscle patterning to affective imagery in depressed and nondepressed subjects. Science 1976;192:489-491

26. Tomkins SS. Affect, Imagery, and Consciousness: Vol. I. The Positive Effects. New York, NY: Springer; 1960

27. VanSwearingen JM, Cohn JF, Turnbull J, Mrzai T, Johnson PC. Psychological distress: linking impairment with disability in facial neuromuscular disorders. Otolaryngol Head Neck Surg 1998;118:790-796

28. Merkel KE, Schmidt KL, Levenstein RM, VanSwearingen JM, Bentley BC. Positive affect predicts improved lip movement in facial movement disorder. Otolaryngol Head Neck Surg 2007;137:100-104

29. VanSwearingen JM, Cohn JF. Smiling in facial paralysis. In: Beurskens CHG, vanGelder RS, Heymans PG, Manni JJ, Nicolai JPA, eds. The Facial Palsies: Complementary Approaches. Utrecht, The Netherlands: LEMMA Publishers; 2005:373-386

30. VanSwearingen JM, Cohn JF, Bajaj-Luthra A. Specific impairment of smiling increases the severity of depressive symptoms in patients with facial neuromuscular disorders. Aesthetic Plast Surg 1999;23:416-423

31. MacGregor FC. Facial disfigurement: problems and management of social interaction and implications for mental health. Aesthetic Plast Surg 1990;14:249-257

32. Byrne PJ. Importance of facial expressio in facial nerve rehabilitation. Curr Opin Otolaryngol Head Neck Surg 2004;12:332-335

33. Schmidt KL, VanSwearingen JM, Levenstein RM. Speed, amplitude, and asymmetry of lip movement in voluntary puckering and blowing expressions: implications fo facial assessment. Motor Control 2005;9:270-280

34. Waxman B. Electrotherapy for treatment of facial paralysis (Bell's palsy). Health Technology Assessment Reports. 3rd ed. National Centre for Health Services Research, US Dept of Health; 1984:27
35. Miehlke A, Stennert E, Chilla R. New aspects in facial narve surgery. Clin Plast Surg 1979;6:451-470

36. Mosforth J, Taverner D. Physiotherapy for Bell's palsy. BMJ 1958;2:675-677

37. Jansen JK, Lomo T, Nicolaysen K, Westgaard RH. Hyperinnervation of skeletal muscle fibers: dependence on muscle activity. Science 1973;181:559-561

38. Cohan CS, Kater SB. Suppression of neurite elongation and growth cone motility by electrical activity. Science 1986;232: 1638-1640

39. Brown MC, Holland RL. A central role for denervated tissues in causing nerve sprouting. Nature 1979;282:724726

40. Farragher D, Kidd GL, Tallis R. Eutrophic electrical stimulation for Bell's palsy. Clin Rehabil 1987;1:265271

41. Cole J, Zimmerman S, Gerson S. Nonsurgical neuromuscular rehabilitation of facial muscle paresis. In: Rubin LR, ed. The Paralyzed Face. St. Louis, MO: Mosby; 1991:107-112

42. Balliet R. Facial paralysis and other neuromuscular dysfunctions of the peripheral nervous system. In: Payton OD, ed. Manual of Physical Therapy Techniques. New York, NY: Churchill Livingston; 1989:41-76

43. Moldaver J. Mimetic muscles and variations of expressions. In: Moldaver J, Conley J, Brown D, eds. The Facial Palsies: Their Physiopathology and Therapeutic Approaches. Springfield, IL: Charles C. Thomas Books; 1980:11-19

44. Segal B, Hunter T, Danys I, Freedman C, Black M. Minimizing synkinesis during rehabilitation of the paralyzed face; preliminary assessment of a new small-movement therapy. J Otolaryngol 1995;24:149-153

45. Balliet R, Shinn JB, Bach-y-Rita P. Facial paralysis rehabiitation: retraining selective muscle control. Int Rehabil Med 1982;4:67-74

46. Brach JS, VanSwearingen J, Delitto A, Johnson PC. Impairment and disability in patients with facial neuromuscular dysfunction. Otolaryngol Head Neck Surg 1997; 117:315-321

47. VanSwearingen JM, Brach JS. Validation of a treatmentbased classification system and tailored treatment approach for individuals with facial neuromotor disorders. Phys Ther 1998;78:678-689

48. Delitto A, Erhard RE, Bowling RW. A treatment-based classification approach to low back syndrome: Identifying and staging patients for conservative treatment. Phys Ther 1995; 75:470-489

49. Jette AM. Diagnosis and classification by physical therapists: a special communication. Phys Ther 1989;69:967-969

50. Buchbinder R, Goel V, Bombardier C, Hogg-Hohnson S. Classification systems of soft tissue disorder of the neck and upper limb: Do they satisfy methodological guidelines? J Clin Epidemiol 1996;49:141-149

51. Sackett DL, Haynes RB, Guyatt GH, Tugwell P. Clinical Eipdemiology. Second ed. Boston, MA: Little Brown; 1991

52. Andersen RG, Newsom HT. Facial nerve disorders. Selected Readings Plastic Surgery. 1987;4:1-28

53. Borodic GE, Cozzolino D. Blepharospasm and its treatment, with emphasis on the use of botulinum toxin. Plast Reconstr Surg 1989;83:546-554

54. May M. Facial nerve disorders. Am J Otol 1982;4:77-87

55. May M, Croxson GR, Klein SR. Management of sequalae using EMG rehabilitation, botulinum toxin and surgery. Am J Otol 1989;10:220-229 
56. Brook RH, Kamberg CJ. General health status measures and outcome measurement: a commmentary on measuring functional status. J Chronic Dis 1987;1:131-165

57. World Health Organization. International Classification of Impairments, Disabilities, and Handicaps. Geneva, Switzerland: World Health Organization; 1980

58. Ross BG, Fradet G, Nedzelski JM. Development of a sensitive clinical facial grading system. Otolaryngol Head Neck Surg 1996;114:380-386

59. VanSwearingen JM, Brach JS. The facial disability index: reliability and validity of a disability assessment instrument for disorders of the facial neuromuscular system. Phys Ther 1996;76:1288-1300

60. Ware JE, Sherbourne CD. The MOS 36-item short-form health survey (SF-36). Med Care 1992;30:473-483

61. Jacobson E. Progressive Relaxation. Second ed. Chicago, IL: University of Chicago Press; 1938

62. Ekman P, Friesen WV. Facial Action Coding System. Palo Alto, CA: Consulting Psychological Press; 1978

63. Ekman P. Symposium on emotion. Facial expressions of emotion: new findings, new questions. Psychol Sci1992;3:34-38
64. Levenson RW, Ekman P, Friesen WV. Voluntary facial action generates emotion-specific autonomic nervous system activity. Psychophysiology 1990;27:363-384

65. Bender MB, Bruyn GW, Vinken PJ. Disorders of eye movements. In: Vinken, Bruyn, eds. Handbook of Clinical Neurology. Amsterdam, The Netherlands: North-Holland; 1969:574-630

66. Bender MB. Comments on the physiology and pathology of eye movements in the vertical plane. J Nerv Ment Dis 1960; 130:156-166

67. Esteban A, Salinero E. Reciprocal reflex activity in ocular muscels: implications in spontaneous blinking and Bell's phenomenon. Eur Neurol 1979;18:157-165

68. VanSwearingen J, Henkelmann TC, Wachtman GS, Manders EK, Cohn JF. Evidence for neuromuscular reeducation of eye closure in persons with facial palsy. (Abstract) presented at: Science 2005, University of Pittsbugh, Pittsbugh, PA, 2005

69. Brooks VB. The Neural Basis of Motor Control. New York, NY: Oxford University Press; 1986 